\title{
Experiments and Potentialities for the use of Bessel Beam in Superresolution STEM
}

\author{
Vincenzo Grillo ${ }^{1,2}$, Ebrahim Karimi ${ }^{3}$, Roberto Balboni ${ }^{4}$, Gian Carlo Gazzadi ${ }^{1}$, Stefano Frabboni ${ }^{1,5}$ Erfan \\ Mafakheri $^{1,5}$ and Robert W. Boyd ${ }^{3,6}$ \\ 1. CNR-Istituto Nanoscienze, Centro S3, Via G Campi 213/a, I-41125 Modena, Italy \\ 2. CNR-IMEM, Parco delle Scienze 37a, I-43100 Parma, Italy. \\ 3. Department of Physics, University of Ottawa, Ottawa, Ontario K1N 6N5, Canada \\ 4. CNR-IMM Bologna, Via P. Gobetti 101, 40129 Bologna, Italy \\ 5. Dipartimento FIM, Universitá di Modena e Reggio Emilia, Via G. Campi 213/a, 41125 Modena, Italy \\ 6. Institute of Optics, University of Rochester, Rochester, New York 14627, USA
}

In light optics holographic beam shaping has been largely used to obtain complicated wavefronts [1] and vortex beams. In the last years holograms have been used in the electron vortex beam generation [2]. We have recently improved the technology of hologram fabrication by means of "phase hologram" [3] replacing the amplitude hologram so far used. Phase holograms appropriately change the phase of the incident wavefront by a modulation of thickness in a silicon nitride thin membrane. Beyond vortex generation, holograms can have a large range of applications. As an example in this work we report on the production of a Bessel beam in the Fresnel diffraction regime [4].

Bessel beams have many interesting properties most of which derived from being the Fourier transform of a ring. In this sense they can be considered as the extreme case of hollow cone illumination. However while the production of hollow cone probes requires a strong reduction of the electron beam current by the use of an obstructing aperture, the holographic approach permits to produce high quality Bessel beams with only marginal intensity losses. In particular by the use of phase holograms we could demonstrate up to $40 \%$ efficiency. In these conditions the generated beam is competitive with normal aperture limited approaches in terms of intensity.

Fig 1a shows the FIB-nanofabricated hologram that has been positioned in the second condenser aperture of a FEI Tecnai F20 operated at $200 \mathrm{kV}$. Fig $1 \mathrm{~b}$ is an image of the logarithm of the intensity of the Bessel beam showing the characteristic fringes aside from the central peak. The obtained probe size is in this case $0.5 \mathrm{~nm}$. But we will show that the beam can be potentially scaled to $0.1 \mathrm{~nm}$ for larger convergences. In Fig 1c the Fraunhofer diffraction of the hologram is also shown demonstrating that the probe is the Fourier transform of a tiny ring. The calculated convergence is here 1.9 mrad.

This beam can be exploited in many applications. One of the main advantages of this is that the probe shape is, to large extent, independent from spherical aberration, chromatic aberration and from defocus; the other is that for the given convergence Bessel beam provide the minimal probe size regardless of spherical aberration.

Fig $2 \mathrm{a}$ shows the transfer function of a microscope with a conventional probe (with and without aberration) with convergence $15 \mathrm{mrad}$ and with a Bessel probe. Clearly the Bessel probe can produce advantages in the high frequency region. Fig $2 b$ is a simulation of STEM-HAADF images for a Au particle with the two kinds of probes as in fig 2a. Using the Bessel beams in fig 1a we obtained the STEM image in fig 2c that demonstrates that, in spite of the presence of other diffraction peaks, a good quality scan of a sample (a Si-SiO STI structure) can be obtained with a resolution better than $2 \mathrm{~nm}$ (measured as the blurring of the contrast features). In fact the transmitted and other diffraction beam are completely delocalized and do not contribute significantly to the contrast. 
Moreover using a deconvolution method [5] we could get an effective resolution $\mathrm{d}<1 \mathrm{~nm}$ demonstrating that the effect of the long tails of the Bessel beam can be partially reduced through numerical procedures.

\section{References:}

[1] V. Y. Bazhenov, M. V. Vasnetsov, and M. S. Soskin, JETP Lett. 52, 429 (1990).

[2] J. Verbeeck, H. Tian, and P. Schattschneider, Nature 467, 301 (2010).

[3] V. Grillo G.C. Gazzadi, E. Karimi et al. Applied Physics Letters 104, 043109 (2014)

[4] V. Grillo, E. Karimi, G.C. Gazzadi et al Physical Review X 4, 011013 (2014)

[5] V. Grillo and F. Rossi Ultramicroscopy 125 (2013) 112

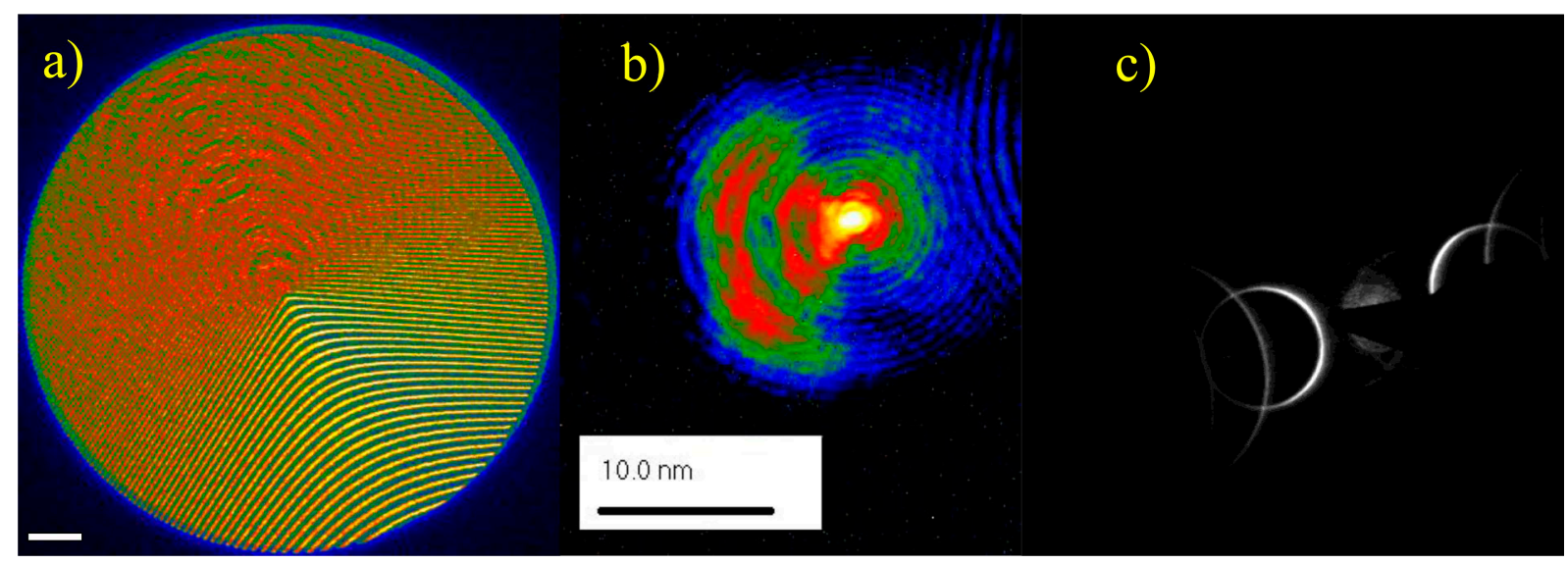

Figure 1. a) TEM image of the hologram b) image of a Bessel beam (logarithmic scale) in the Fresnel plane c) Diffraction pattern of the aperture: each circle corresponds to an order of the hologram diffraction.
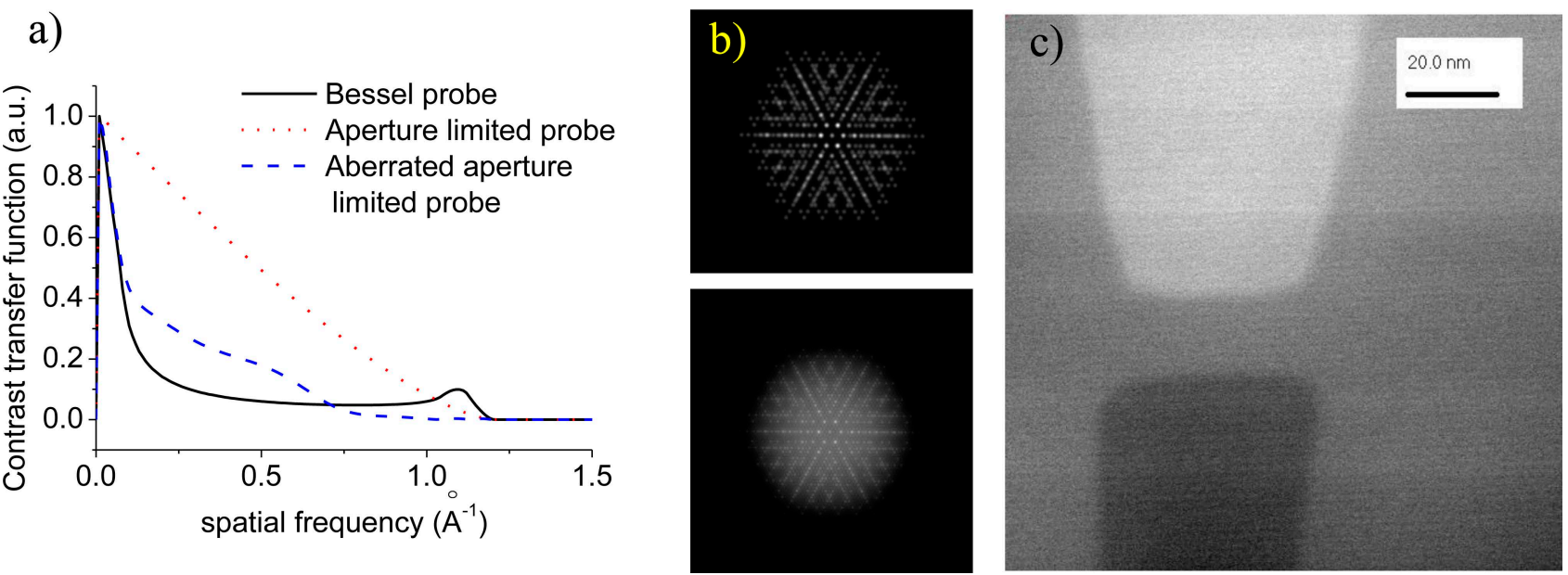

Figure 2. a) Calculation of the contrast transfer function for a Bessel beam (solid), for aperture limited beam with no aberration (dot) and with aberrations (dash) $\mathrm{Cs}=0.5 \mathrm{~mm}$ defocus $40 \mathrm{~nm}$. In all cases the convergence was $15 \mathrm{mrad}$. b) Simulation of a STEM-HAADF of a Au particle for an aperture limited (top) and Bessel (bottom) beam c) experimental STEM image using the Bessel probe as in fig 1a. 